\title{
Evaluación de Lesiones Periapicales en Pacientes Derivados a Cirugía Periapical Mediante Tomografía Computarizada de Haz Cónico
}

\author{
Evaluation of Periapical Lesions by Cone Beam Computed \\ Tomography in Patients Referred for Apical Surgery
}

\author{
Scarlette Hernández Vigueras"; Manuel Donoso Zúñiga²; Claudio Sanhueza Tobar; \\ Jared Linco Olave ${ }^{4} \&$ Sebastián Riquelme Carrasco
}

HERNÁNDEZ, V. S.; DONOSO, Z. M.; SANHUEZA, T. C.; LINCO, O. J. \& RIQUELME, C. S. Evaluación de lesiones periapicales en pacientes derivados a cirugía periapical mediante tomografía computarizada de haz cónico. Int. J. Odontostomat., 11(2):128-132, 2017.

RESUMEN: El uso de la tomografía computarizada de haz cónico (CBCT) en endodoncia ha ido en aumento, justificando su uso en casos más complejos, como cirugías periapicales. El objetivo fue evaluar las características imagenológicas encontradas en un grupo de pacientes derivados a cirugía periapical, mediante el uso de CBCT. Se realizó un estudio observacional descriptivo, en un grupo de pacientes, derivados a cirugía periapical, a los cuales se realizó un examen de CBCT, evaluación clínica y radiografía periapical. Un total de 18 pacientes fueron examinados (6 hombres y 12 mujeres), cuyas edades estaban entre los 19 y 64 años de edad. El diámetro mayor de las lesiones varió entre 6 mm a 16 $\mathrm{mm}$. El uso de CBCT en cirugías periapicales entrega información más completa al cirujano, ya que permite mayor precisión al realizar el acceso quirúrgico y detecta un mayor número de lesiones periapicales que con la radiografía periapical, logrando en casos complejos un mejor diagnóstico y planificación del tratamiento.

PALABRAS CLAVE: tomografía computarizada de haz cónico, cirugía periapical, endodoncia.

\section{INTRODUCCIÓN}

En pacientes con indicación de cirugía periapical, es esencial realizar una exhaustiva historia clínica, un adecuado examen clínico y radiográfico, apoyados principalmente en exámenes imagenológicos. (American Association of Endodontists \& American Academy of Oral and Maxillofacial Radiology, 2011).

Convencionalmente se ha utilizado la radiografía periapical, la cual permite el análisis de estructuras periapicales, entregando una visión más específica de un diente o región en particular (Huumonen \& Ørstavik, 2002), presentando una alta sensibilidad, principalmente en la región anterior (Georgopoulou et al., 2005). Sin embargo, su uso brinda información limitada ya que sólo es una representación bidimensional de estructuras tridimensionales. La superposición de estructuras anatómicas adyacentes, como la sobreproyección de las raíces o la presencia del seno maxilar, aumenta la dificultad diagnóstica (Low et al., 2008). La distorsión en radiografías periapicales, puede generar un aumento o disminución del tamaño de la lesión, resultando en ocasiones, en la incapacidad para visualizarlas (Lofthag-Hansen et al., 2007). Por lo cual, existen diferentes factores que reducen la capacidad diagnóstica de la radiografía periapical (Scarfe et al., 2009).

La Tomografía Computarizada de Haz Cónico (CBCT) es un sistema de diagnóstico imagenológico, contem-

\footnotetext{
${ }^{1}$ Especialista en Endodoncia, Doctor en Ciencias Odontológicas. Instituto de Odontoestomatología, Facultad de Medicina, Universidad Austral de Chile, Valdivia, Chile.

${ }^{2}$ Patólogo Oral, Facultad de Medicina, Universidad Austral de Chile. Hospital Base Valdivia, Chile.

${ }^{3}$ Radiólogo Oral y Maxilofacial, Universidad San Sebastián, Valdivia, Chile.

${ }^{4}$ Escuela de Odontología, Universidad Austral de Chile, Valdivia, Chile.
} 
poráneo, de visualización tridimensional, diseñado especialmente para su uso en el esqueleto maxilofacial, siendo significativamente más sensible que la radiografía convencional a la detección de periodontitis apicales en humanos (Durack \& Patel, 2012). Con su uso se pueden detectar lesiones adicionales, reportándose que en un $70 \%$ de los casos entrega información clínicamente relevante, que no se encontraba con el uso de radiografía periapical (Lofthag-Hansen et al.). Adicionalmente, puede identificar destrucción periapical ósea asociada con la infección endodóntica, antes de que haya evidencia de su existencia en las radiografías convencionales (Durack \& Patel).

La Asociación Americana de Endodoncia (AAE) y la Academia Americana de Cirugía Oral y Radiología Maxilofacial (AAOMR), han entregado su posición con respecto al uso de CBCT en endodoncia, con la intención de proporcionar una orientación científica a los clínicos sobre su uso como complemento en tratamientos endodónticos complejos (American Association of Endodontists \& American Academy of Oral and Maxillofacial Radiology).

El objetivo de este estudio fue describir las características imagenológicas encontradas en un grupo de pacientes derivados a cirugía periapical, mediante el uso de CBCT.

\section{MATERIAL Y MÉTODO}

Para la realización del estudio se contó con la aprobación del Comité de Ética del Servicio de Salud Valdivia ( $\left.\mathrm{N}^{\circ} 231\right)$, Chile.

Se seleccionaron pacientes derivados para la realización de cirugía periapicales en el Hospital Base de Valdivia, Chile, durante el año 2012-2013.

El criterio de inclusión utilizado fue de incorporar pacientes derivados a cirugía paraendodóntica dentro de este establecimiento, dientes a rehabilitarse dentro del mismo sistema de atención, con adecuado soporte periodontal, y que hayan recibido tratamiento o retratamiento de endodoncia, en caso de ser necesario, antes de ser derivado a cirugía. Se excluyeron de está investigación aquellos pacientes que no aceptaran participar, o pacientes en cuyos exámenes presentarán imágenes borrosas y/o artefactos que impidan la visualización del diente en estudio.
Los pacientes que cumplían con los criterios de selección, se les informó claramente las características del estudio y sus objetivos, los cuales firmaron el consentimiento informado para participar. Estos fueron derivados con la respectiva radiografía periapical para la programación de la cirugía, a los cuales se les citó adicionalmente, para realizar un CBCT en un centro odontológico privado de la ciudad, sin costo asociado para el paciente.

Todos los exámenes fueron realizados por un especialista en radiología bucomaxilofacial. Las adquisiciones de CBCT fueron obtenidas con un equipo Sky View (MyRay, Imola, Italia 2009), de 12 bits utilizando parámetros de exposición de $90 \mathrm{Kv}$ y $10 \mathrm{~mA}$. Para los estudios maxilares se utilizó un FOV de $7 \mathrm{~cm}$ con un voxel de 0,17 mm, en el caso de los dientes mandibulares se usó un FOV de $11 \mathrm{~cm}$ y un voxel de $0,22 \mathrm{~mm}$. Como protocolo diagnóstico los volúmenes fueron previamente alineados siguiendo como guía en maxilar el piso de fosas nasales y el eje de implantación de los premolares. En mandíbula, se utilizó como referencia el borde basilar y eje de implantación de los premolares. Además, se trazaron las curvas necesarias para realizar reconstrucciones panorámicas y los cortes transversales respectivos. El observador realizó el análisis de los exámenes $\mathrm{CBCT}$ mediante la visualización de la reconstrucción panorámica con diferentes espesores, observación de la reconstrucción 3D con distintos filtros disponibles, la interpretación diagnóstica de los cortes transversales se realizó con $1 \mathrm{~mm}$ de espesor y $0,5 \mathrm{~mm}$ de separación entre cortes, y la visualización del volumen en los tres planos del espacio, utilizando reconstrucciones multiplanares (MPR). Se informó las dimensiones de las lesiones apicales asociadas a los dientes que requerían cirugía periapical, compromiso de tablas óseas bucales o palatinas/linguales, y otros hallazgos de interés observados en el examen. Se registraron la cantidad de dientes involucrados en la lesión, el mayor diámetro de ésta, analizándola en tres dimensiones, mesiodistal, bucolingual y coronoapical.

\section{RESULTADOS}

De los 18 pacientes seleccionados, 6 eran hombres y 12 mujeres, con un rango de edades entre los 19 y 64 años de edad. Del total de pacientes, 7 presentaban antecedentes de enfermedades sistémicas, hipertensión $(n=4)$, diabetes $(n=2)$, hipotiroidismo $(n=1)$ y epilepsia $(n=2)$. 
Los diagnósticos clínicos más frecuente fueron Absceso Dentoalveolar Crónico $(n=8)$ y Periodontitis Apical Sintomática $(n=8)$, según criterios propuestos por la Asociación Americana de Endodoncia 2009.

Las lesiones periapicales involucraban un total de
23 dientes, de los cuales, 16 corresponden a dientes anterosuperiores, cuatro dientes anteroinferiores y tres a premolares superiores.

El diámetro mayor de las lesiones varió entre $6 \mathrm{~mm}$ a $16 \mathrm{~mm}$, el cual tuvo una media 9,6 mm. (Figs 1 y 2).

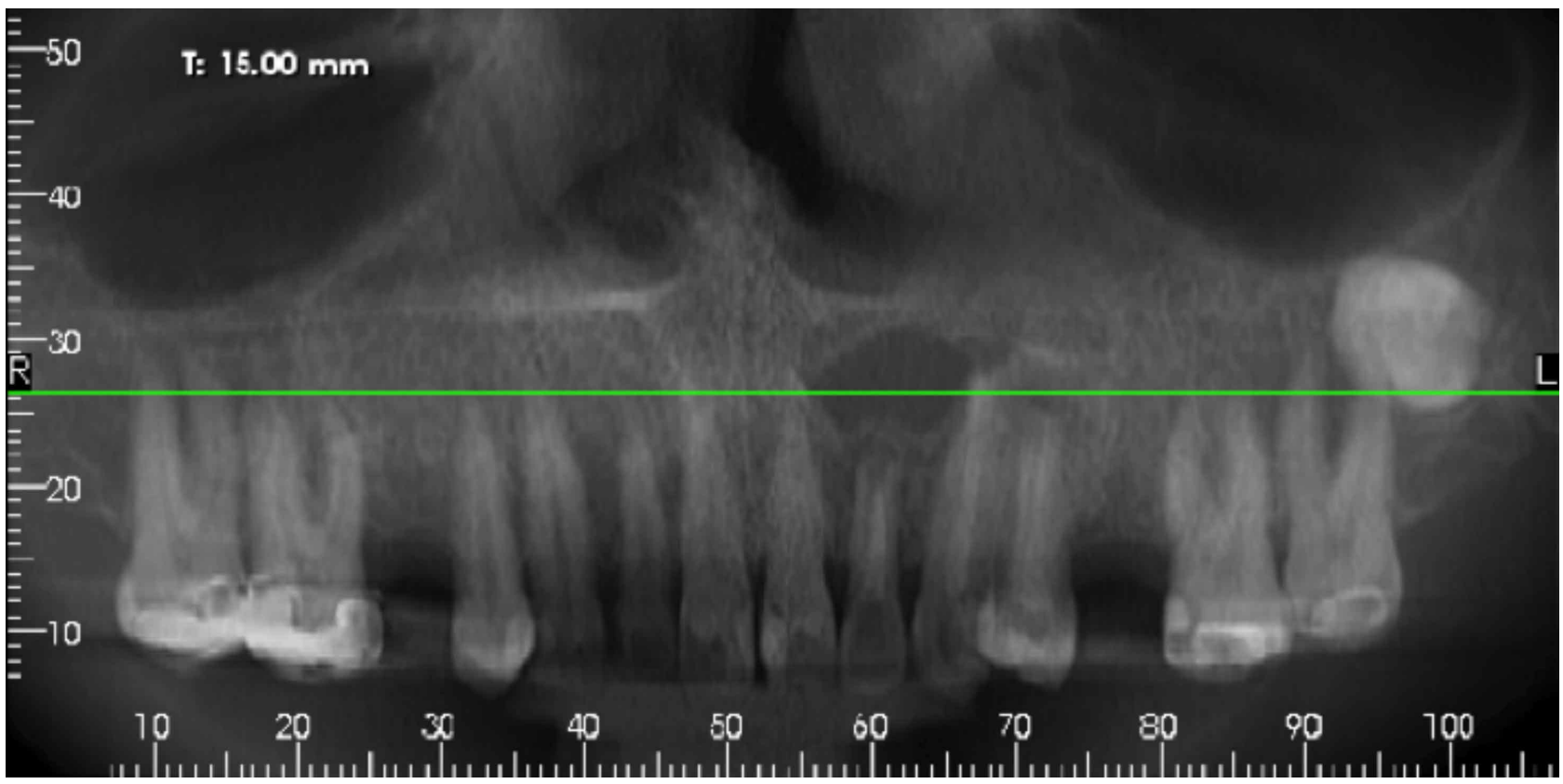

Fig. 1. Reconstrucción panorámica del CBCT, mostrando aspectos similares a los entregados en una radiografía panorámica.

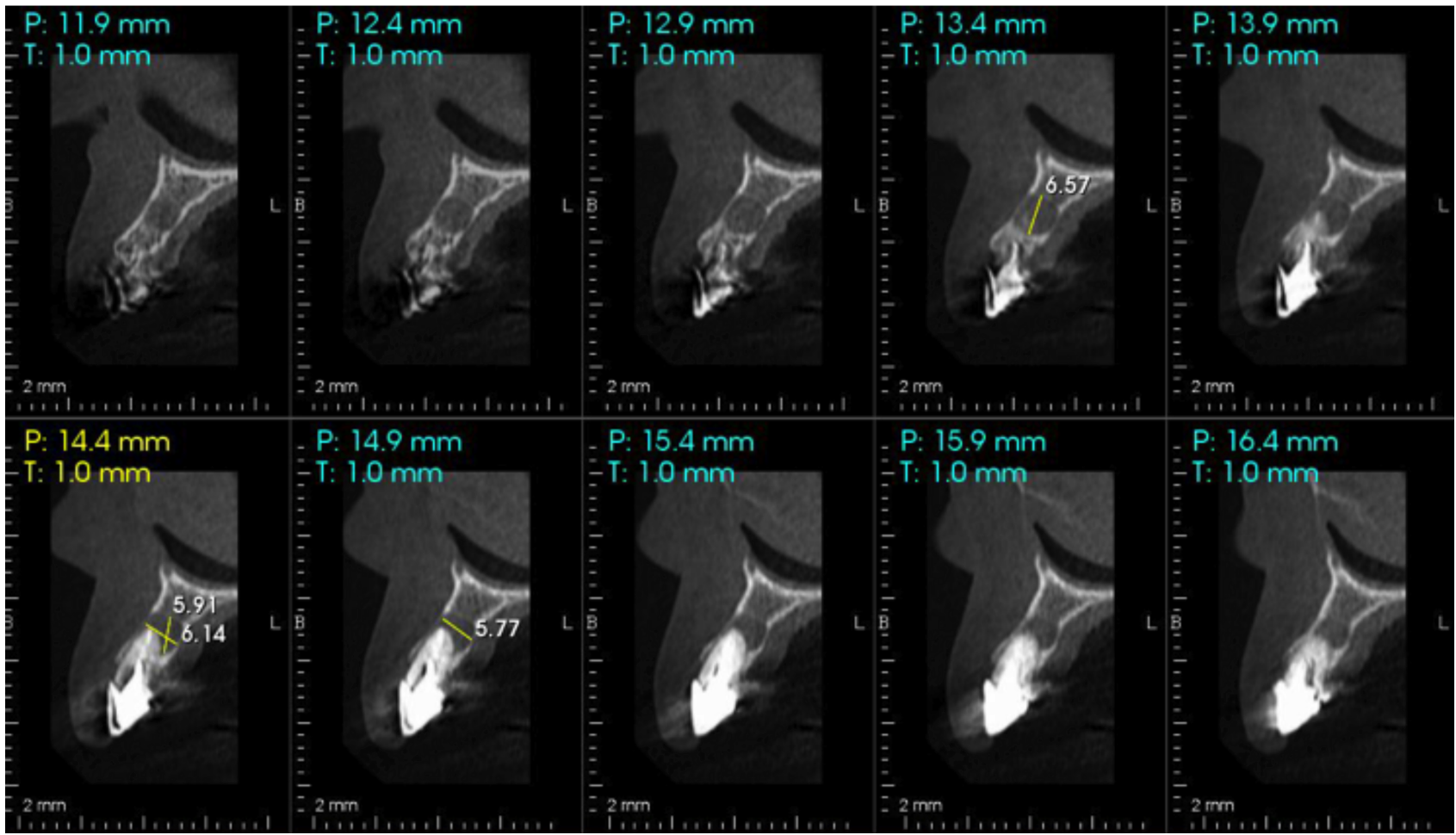

Fig. 2 Secuencia de cortes tranversales de $1 \mathrm{~mm}$ de espesor con un intervalo de $0.5 \mathrm{~mm}$ entre los cortes del estudio CBCT. 
HERNÁNDEZ, V. S.; DONOSO, Z. M.; SANHUEZA, T. C.; LINCO, O. J. \& RIQUELME, C. S. Evaluación de lesiones periapicales en pacientes derivados a cirugía periapical mediante tomografía computarizada de haz cónico. Int. J. Odontostomat., 11(2):128-132, 2017.

\section{DISCUSIÓN}

Con respecto a la identificación de lesiones periapicales, Low, concluyó que el uso de CBCT detecta un $34 \%$ más de lesiones que no eran observables en radiografías periapicales en premolares y molares maxilares (Low et al.). Otro estudio demostró que CBCT detectó lesiones periapicales en 37 raíces $(25,9 \%)$, en comparación con $18(12,6 \%)$ de las radiografías periapicales (Liang et al., 2011). Estrela et al. (2008) evaluaron la precisión diagnóstica de lesiones apicales en radiografías panorámicas y periapicales en comparación a CBCT, reportando los valores de sensibilidad, especificidad, valores predictivos positivos y negativo; se evaluaron a 888 pacientes (1508 dientes) concluyendo que las radiografías periapicales tienen un alto valor predictivo positivo, sin embargo, presentan un bajo valor predictivo negativo, es decir, en un $55 \%$ de las radiografías periapicales corresponden a situaciones donde realmente no hay lesiones.

El uso de CBCT es recomendado en la planificación de cirugías periapicales, ya que permite evaluar el verdadero alcance de las lesiones y su relación espacial con estructuras anatómicas importantes, como el canal mandibular, el seno maxilar y el foramen mental (Patel, 2009). En el diagnóstico inicial y en la planificación pre-quirúrgica, la exploración con CBCT permite el diagnóstico diferencial con otras lesiones de origen no endodóntico, tales como quistes y tumores odontogénicos y no odontogénicos (Faitaroni et al., 2011). Adicionalmente, el CBCT puede usarse para medir los valores en la escala de grises, lo cual puede entregar un método más exacto en las lesiones periapicales para diferenciar el contenido sólido o una cavidad llena de líquido (Simon et al., 2006).

La precisión geométrica de CBCT se ha confirmado en varios estudios, lo cual permite evaluar con exactitud el tamaño, naturaleza y posición de las lesiones periapicales (Lofthag-Hansen et al.; Patel). Los voxels de CBCT al ser isotrópicos, es decir, iguales en altura, longitud y profundidad, permiten una medición geométrica más exacta en cualquier plano evaluado (Patel). Liang et al. (2014) concluyeron que la medición volumétrica con CBCT fue más precisa que con radiografías periapicales convencionales en el diagnóstico de lesiones periapicales de dientes inferiores.

Una de las mayores ventajas del CBCT sobre la TAC es la menor dosis de radiación efectiva a la cual es sometido el paciente. Los CBCT de volumen limitado ofrecen una dosis efectiva menor y son los más adecuados para casos clínicos de endodoncia proporcionando imágenes de varios dientes con aproximadamente la misma radiación de dos radiografías periapicales y reduciendo la radiación cuando se realizan múltiples imágenes tradicionales en casos complejos (Ludlow et al., 2006).

El mayor problema que afecta a la calidad de la imagen y la precisión diagnóstica de imágenes con CBCT es la dispersión y endurecimiento de haz de rayos, debido a la alta densidad de estructuras, como postes de metal y restauraciones, reduciendo en estos casos, el valor diagnóstico en la evaluación del diente con lesiones periapicales (American Association of Endodontists \& American Academy of Oral and Maxillofacial Radiology; Lofthag-Hansen et al.).

Por lo tanto, el uso de CBCT debe considerarse como un complemento para las radiografías periapicales convencionales. Su capacidad para mostrar imágenes geométricamente precisas en tres dimensiones, la eliminación de ruido anatómico adyacente, la sensibilidad y la dosis efectiva comparable, confieren ventajas importantes para el diagnóstico, evaluación y planificación quirúrgica de lesiones periapicales.

AGRADECIMIENTOS. Fondos de la Dirección de Investigación y Desarrollo, Universidad Austral de Chile, Chile, Proyecto DID S-2012-05.

HERNÁNDEZ, V. S.; DONOSO, Z. M.; SANHUEZA, T. C.; LINCO, O. J. \& RIQUELME, C. S. Evaluation of periapical lesions by cone beam computed tomography in patients referred for apical surgery. Int. J. Odontostomat., 11(2):128132, 2017.

ABSTRACT: The use of cone beam computed tomography (CBCT) in endodontics has increased, justifying its use in more complex cases, such as apical surgery. The aim was to evaluate the radiologic characteristics found in a group of patients referred to apical surgery, using CBCT. A descriptive study was conducted in a group of patients referred for apical surgery. In these patients, a clinical examination, a conventional radiographic evaluation and CBCT were performed. A total of 18 patients were examined 
( 6 men and 12 women), ages were between 19 and 64 years old. The major diameter of the lesions ranged from $6 \mathrm{~mm}$ to $16 \mathrm{~mm}$. The use of CBCT in apical surgery provides more complete information to the surgeon, allowing greater precision when performing surgical access and detects a greater number of periapical lesions than with conventional radiography. In complex cases is possible obtain better diagnosis and treatment planning.

KEY WORDS: cone beam computed tomography, endodontic, apical surgery.

\section{REFERENCIAS BIBLIOGRÁFICAS}

American Association of Endodontists \& American Academy of Oral and Maxillofacial Radiology. Use of cone-beam computed tomography in endodontics Joint Position Statement of the American Association of Endodontists and the American Academy of Oral and Maxillofacial Radiology. Oral Surg. Oral Med. Oral Pathol. Oral Radiol. Endod., 111(2):234-7, 2011.

Durack, C. \& Patel, S. Cone beam computed tomography in endodontics. Braz. Dent. J., 23(3):179-91, 2012.

Estrela, C.; Bueno, M. R.; Leles, C. R.; Azevedo, B. \& Azevedo, J. R. Accuracy of cone beam computed tomography and panoramic and periapical radiography for detection of apical periodontitis. J. Endod., 34(3):273-9, 2008.

Faitaroni, L. A.; Bueno, M. R.; Carvalhosa, A. A.; Mendonça, E. F. \& Estrela, C. Differential diagnosis of apical periodontitis and nasopalatine duct cyst. J. Endod., 37(3):403-10, 2011.

Georgopoulou, M. K.; Spanaki-Voreadi, A. P.; Pantazis, N. \& Kontakiotis, E. G. Frequency and distribution of root filled teeth and apical periodontitis in a Greek population. Int. Endod. J., 38(2):105-11, 2005.

Huumonen, S. \& Ørstavik, D. Radiological aspects of apical periodontitis. Endod. Top., 1(1):3-25, 2002.

Lofthag-Hansen, S.; Huumonen, S.; Gröndahl, K. \& Gröndahl, H. G. Limited cone-beam CT and intraoral radiography for the diagnosis of periapical pathology. Oral Surg. Oral Med. Oral Pathol. Oral Radiol. Endod., 103(1):114-9, 2007.

Low, K. M.; Dula, K.; Bürgin, W. \& von Arx, T. Comparison of periapical radiography and limited cone-beam tomography in posterior maxillary teeth referred for apical surgery. J. Endod., 34(5):55762, 2008.

Liang, Y. H.; Li, G.; Wesselink, P. R. \& Wu, M. K. Endodontic outcome predictors identified with periapical radiographs and cone-beam computed tomography scans. J. Endod., 37(3):326-31, 2011.

Liang, Y. H.; Jiang, L.; Gao, X. J., Shemesh, H.; Wesselink, P. R. \& $\mathrm{Wu}, \mathrm{M}$. K. Detection and measurement of artificial periapical lesions by cone-beam computed tomography. Int. Endod. J., 47(4):332-8, 2014

Ludlow, J. B.; Davies-Ludlow, L. E.; Brooks, S. L. \& Howerton, W. B. Dosimetry of 3 CBCT devices for oral and maxillofacial radiology: CB Mercuray, NewTom $3 \mathrm{G}$ and i-CAT. Dentomaxillofac. Radiol., 35(4):219-26, 2006.

Patel, S. New dimensions in endodontic imaging: Part 2. Cone beam computed tomography. Int. Endod. J., 42(6):463-75, 2009.

Scarfe, W. C.; Levin, M. D.; Gane, D. \& Farman, A. G. Use of cone beam computed tomography in endodontics. Int. J. Dent., 2009: 634567, 2009.

Simon, J. H.; Enciso, R.; Malfaz, J. M.; Roges, R.; Bailey-Perry, M. \& Patel, A. Differential diagnosis of large periapical lesions using cone-beam computed tomography measurements and biopsy. J. Endod., 32(9):833-7, 2006.
Dirección para correspondencia:

Scarlette Hernández Vigueras

Instituto de Odontoestomatología

Universidad Austral de Chile

Rudloff 1640

Valdivia

CHILE

E-mail: shernandez@uach.cl

Recibido : 06-10-2016

Aceptado: 09-01-2017 